\title{
Malaria research challenges in low prevalence settings
}

\author{
Gillian Stresman', Tamaki Kobayashi ${ }^{2}$, Aniset Kamanga ${ }^{3}$, Philip E Thuma ${ }^{4}$, Sungano Mharakurwa ${ }^{4,5}$, \\ William J Moss ${ }^{2}$ and Clive Shiff ${ }^{*}$
}

\begin{abstract}
The prevalence of malaria has reduced significantly in some areas over the past decade. These reductions have made local elimination possible and the research agenda has shifted to this new priority. However, there are critical issues that arise when studying malaria in low transmission settings, particularly identifying asymptomatic infections, accurate detection of individuals with microparasitaemic infections, and achieving a sufficient sample size to have an adequately powered study. These challenges could adversely impact the study of malaria elimination if they remain unanswered.
\end{abstract}

\section{Background}

After the failed eradication attempt of the 1950s, the malaria research objective was clear: reduce morbidity and mortality rates in areas where malaria persisted. These objectives made malaria relatively easy to study as areas with high morbidity and mortality had a high prevalence of infection as well as high parasite densities within infected individuals [1-4]. The focus on mortality reduction led to the development and widespread use of important tools to fight malaria, such as insecticide-treated bed nets, rapid diagnostic tests, and artemisinin, a drug effective against asexual parasitaemia and immature gametocytes, all of which have likely contributed to the reductions in malaria transmission in some areas [5-7]. The dramatic decline in malaria transmission has led to a shift from control to elimination as the primary goal [8]. This new malaria research agenda has led to new challenges: the populations of interest now include those that experience a low prevalence of infection and consist of asymptomatic and microparasitaemic individuals that may be beyond the limit of detection with current diagnostic tools. The ability to accurately study populations that are likely responsible for sustaining malaria transmission from one season to the next will be critical to achieving elimination, but are particularly difficult to assess with the diagnostic and epidemiological tools currently available $[9,10]$.

\footnotetext{
* Correspondence: cshiff@jhsph.edu

${ }^{5}$ Department of Molecular Microbiology and Immunology, Johns Hopkins Bloomberg School of Public Health, Baltimore, MD 21205, USA Full list of author information is available at the end of the article
}

Therefore, it is essential to discuss the current methodological barriers to the study of malaria in settings of low parasite prevalence and density, and the potential implications for confirming malaria elimination.

\section{Targeting asymptomatic infections}

In areas where malaria is sustained at low levels or is highly seasonal, asymptomatic or minimally symptomatic reservoirs of infection are critical for maintaining malaria transmission as the parasite can persist in these individuals from one season to the next $[11,12]$. These reservoirs can comprise more than one third of a population [13] and have inherent challenges that make them difficult to identify, including the fact that these individuals do not seek treatment [14]. However, locating asymptomatically infected people and clearing this parasite reservoir will be critical to sustainable local and national malaria elimination $[15,16]$. In addition to finding ways to detect and prevent importation of malaria, as well as effective vector control measures, if control programmes ignore the local parasite reservoirs in human population's malaria will likely resurge once control efforts are scaled back. A strategy that is simple and cost-effective in targeting gametocyte reservoirs is needed that can easily be integrated into local malaria control programmes.

Malaria infections tend to cluster in geographically defined areas with a higher malaria transmission, or hotspots of infection, during the low transmission season. These clusters that likely serve as the source of parasites 
that fuel subsequent transmission seasons [11]. The concept of hotspots could be used to target parasite reservoirs effectively and achieve maximum impact for control programmes. For example, research conducted in Zambia demonstrated that RDT-confirmed cases of malaria presenting at health facilities during the low transmission season could be used to target reservoirs of asymptomatic infection [17]. However, this approach may lead to sub-optimal coverage and may not be an efficient strategy in areas of low transmission intensity [18]. Other possible methods to target reservoirs could include using school surveys to identify possible hotspots of infection in the community or adults as a sentinel population [19]. If the defining characteristics of a hotspot of malaria infection could be clearly identified using either climate, entomological, or geographical data, these indicators could serve as a powerful tool to inform programmes on where to target control measures [20].

\section{Detection of low levels of parasitaemia}

Even with the existence of a valid method to easily detect hotspots of malaria infection, including asymptomatic populations, a second limitation is our ability to diagnose infected individuals accurately. It is not uncommon for individuals to be infected with parasites that are sustained at microparasitaemic levels. The ability of current diagnostic tools to detect infections when parasite densities are low is poor and individuals negative for malaria by RDT or microscopy are still infectious to mosquitoes. [21] The commonly used tools for malaria diagnosis for research purposes include microscopy, rapid diagnostic tests, measuring the immune response to plasmodium (serology), and various methods of DNA detection including polymerase chain reaction (PCR), loop-mediated isothermal amplification (LAMP), or nucleic acid sequencebased amplification (NASBA) [22]. Although microscopy and RDT are the most commonly used methods of diagnosis, PCR can detect sub- patent infections and is frequently used for research purposes. [23,24]. However, the validity of PCR has not been adequately assessed for asymptomatic or microparasitaemic individuals: with a negative PCR result, what is the probability that a person is truly negative or is the negative due to insufficient sensitivity of the test? The analytical sensitivity of a nested PCR is often cited as being able to detect as few as two parasites per milliliter of whole blood [25]. Although the lower limit of detection is impressive, the consistency of the results in microparasitaemic individuals has never been properly assessed, especially in field settings where sample collection and storage may not be optimum. Microparasitaemic individuals produce gametocytes and likely contribute to the infectious reservoir [14]. However, individuals with infections below the sensitivity of PCR have received little attention and the probability that these individuals will produce and transmit gametocytes to mosquitoes, as well as their contribution to the infectious reservoir, has not been addressed.

\section{Power to detect the effectiveness of interventions}

If it is possible to develop feasible strategies to target asymptomatic populations, and methods exist that can accurately identify those with low levels of parasitaemia, a third challenge provides a more fundamental problem: conducting valid epidemiological studies of malaria elimination in the field. The low transmission season is a critical bottleneck for targeting malaria reservoirs that sustain transmission from one season to the next, but parasite prevalence during this period can be extremely low. [11] Similarly, areas that have reduced transmission can experience extremely low parasite prevalence throughout the year. Both of these circumstances render these regions ideal for elimination campaigns. However, when any disease is uncommon, irrespective of the study design used, epidemiological studies become difficult because of challenges in reaching the desired sample size to achieve adequate power to minimize the probability of wrongly accepting that the null hypothesis is true, known as type II error. For example, a recent clusterrandomized trial carried out in Tanzania to determine the effect of mass drug administration on malaria transmission failed to detect a significant effect between the intervention and control arms because the incidence of malaria was low in the control arm [26].

Achieving the desired sample size and adequate study power is essential for conducting conclusive studies. For some surveys, the challenge of sample size could be overcome by increasing the number of people, increasing the duration of sample collection or survey a broader geographical area. These alternatives come with additional costs and possible challenges of changes in seasonality or variation in the micro epidemiology having an impact on the desired outcome $[27,28]$. Other options include alternative study designs. For example, the use of a pre/post design or the absence of a control population are not common in malaria epidemiology research because traditional epidemiological practice requires a design that allows the relative impact to be measured in reference to a comparison group. However, if malaria is eliminated in a population and the absence of infection confirmed by the absence of malaria antibodies circulating in the blood or other definitive test such as a PCR assay that is both sensitive and demonstrates consistent results, then the effectiveness of the approach would have been demonstrated. The ability to study malaria with sufficient power in elimination settings will be critical to provide insight to the best approaches, their effectiveness and to identify the significance of importations of parasites using molecular epidemiology. 


\section{Implications for malaria elimination}

The presence of asymptomatic infections, the inability to accurately diagnose microparasitaemic infections and gametocytemia, and the difficulty in achieving adequately powered studies in the context of low malaria prevalence all have serious implications for malaria elimination campaigns. How is it possible to understand malaria in low prevalence settings if there is little confidence that the people reported to be negative by PCR are uninfected? If sufficiently powered studies are difficult or impossible to implement, is it possible to eliminate the probability of a null hypothesis? The World Health Organization (WHO) defines malaria as eliminated in an area if there is zero incidence of locally acquired clinical cases in a defined geographical area during three consecutive years [29]. In settings with large numbers of asymptomatically infected people, how can this be assessed without testing populations for serological evidence of recent exposure? Until sufficient measures for elimination are developed for use in areas with persistent pockets of asymptomatic or minimally symptomatically infected individuals, or a successful mass drug administration campaign or vaccine is implemented, how is it possible to claim that these malaria reservoirs are truly eliminated? Also, how perfect does the optimum diagnostic method need to be before there is confidence in the results in an elimination context? In other words, what is the implication for continued transmission if even just one person is misclassified as negative who is actually carrying parasites? For malaria elimination to be sustainable and stand a real chance for success, it is imperative that these fundamental questions in studying malaria in low prevalence settings are addressed while it is still possible for malaria elimination campaigns to succeed.

\section{Competing interests}

The authors declare that they have no conflicts of interest.

\section{Authors' contributions}

GS conceived and prepared the manuscript, and TK, AK, PT, SM, WM, and CS contributed to developing the concepts presented in this paper and provided valuable insight during the revision and editing stages. All authors read and approved the final manuscript.

\footnotetext{
Author details

${ }^{1}$ Department of Immunology and Infection, London School of Hygiene \& Tropical Medicine, London W1CE 7HT, UK. '2Department of Epidemiology, Johns Hopkins Bloomberg School of Public Health, Baltimore, MD 21205, USA. ${ }^{3}$ Faculty of Public Health and Policy, London School of Hygiene \& Tropical Medicine, London W1CE 7HT, UK. ${ }^{4}$ Malaria Institute at Macha, Choma, Zambia. ${ }^{5}$ Department of Molecular Microbiology and Immunology, Johns Hopkins Bloomberg School of Public Health, Baltimore, MD 21205, USA.
}

Received: 6 September 2012 Accepted: 18 October 2012

Published: 25 October 2012

\section{References}

1. Hay SI, Guerra CA, Tatem AJ, Noor AM, Snow RW: The global distribution and population at risk of malaria: past, present, and future. Lancet Infect Dis 2004, 4:327-336.

2. Najera JA, Gonzales-Silva M, Alonso PL: Some lessons for the future from the Global Malaria Eradication Programme (1955-1969). PLoS Med 2011, 8:e1000412.

3. Gething PW, Patil AP, Smith DL, Guerra CA, Elyazar IR, Johnston GL, Tatem AJ, Hay SI: A new world map: Plasmodium falciparum endemicity in 2010. Malar J 2011, 10:378.

4. Shiff C: Integrated approach to malaria control. Clin Microbiol Rev 2002, 15:278-293.

5. Lindsay SW, Snow RW, Broomfield GL, Janneh MS, Wirtz RA, Greenwood BM: Impact of permethrin-treated bedents on malaria transmission by the Anopheles gambiae complex in The Gambia. Med Vet Entomol 1989, 3:263-271.

6. Mharakurwa S, Manyame B, Shiff CJ: Trial of the ParaSight- $F$ test for malaria diagnosis in the primary health care system. Zimbabwe. Trop Med Int Health 1997, 2:544-550.

7. Price RN, Nosten F, Luxemburger C, ter Kulle FO, Paiphun L, Chongsuphajaisiddhi T, White NJ: Effects of artemisinin derivatives on malaria transmissibility. Lancet 1996, 347:1654-1658.

8. Kitua A, Ogundahunsi O, Lines J, Mgone C: Conquering malaria: enhancing the impact of effective interventions towards elimination in the diverse and changing epidemiology. J Glob Infect Dis 2011, 3:161-165.

9. malERA Consultative Group on Diagnoses and Diagnostics: A research agenda for malaria eradication: diagnoses and diagnostics. PLoS Med 2011, 8:e1000396.

10. Manjurano A, Okell L, Lukindo T, Reyburn H, Olomi R, Roper C, Clark TG, Goseph S, Riley EM, Drakeley C: Association of sub-microscopic malaria parasite carriage with transmission intensity in north-eastern Tanzania. Malar J 2011, 10:370.

11. Bousema T, Griffin JT, Sauervwein RW, Smith DL, Churcher TS, Takken W, Ghani A, Drakeley C, Gosling R: Hitting hotspots: spatial targeting of malaria for control and elimination. PLoS Med 2012, 9:e1001165.

12. Harris I, Sharrock WW, Bain LM, Gray KA, Bobogare A, Boaz L, Lilley K, Krause D, Vallely A, Johnson ML, Gatton ML, Shanks GD, Cheng Q: A large proportion of asymptomatic Plasmodium infections with low and submicroscopic parasite densities in the low transmission setting of Temotu Province, Solomon Islands: challenges for malaria diagnostics in an elimination setting. Malar J 2010, 9:254.

13. Baliraine FN, Afrane YA, Amenya DA, Bonizzoni M, Menge DM, Zhou G, Zhong D, Vardo-Zalik AM, Githeko A, Yan G: High prevalence of asymptomatic Plasmodium falciparum infections in a highland area of western Kenya: A cohort study. J Infect Dis 2009, 200:66-74.

14. Manjurano A, Okell L, Lukindo T, Reyburn H, Olomi R, Roper C, Clark TG, Joseph S, Riley EM, Drakeley C: Association of sub-microscopic malaria parasite carriage with transmission intensity in north-eastern Tanzania. Malar J 2011, 10:370

15. Karl S, Gurarie D, Zimmerman PA, King CH, St Pierre TG, Davis TM: A submicroscopic gametocyte reservoir can sustain malaria transmission. PLoS One 2011, 6:e20805.

16. Sutcliffe CG, Kobayashi T, Hamapumbu H, Shields T, Kamanga A, Mharakurwa S, Thuma PE, Glass G, Moss WJ: Changing individual-level risk factors for malaria with declining transmission in southern Zambia: a cross-sectional study. Malar J 2011, 10:324.

17. Stresman GH, Kamanga A, Moono P, Hamapumbu H, Mharakurwa S, Kobayashi T, Moss WJ, Shiff C: A method of active case detection to target reservoirs of asymptomatic malaria and gametocyte carriers in a rural area in Southern Province. Zambia. Malar J 2010, 9:265.

18. Sutcliffe CG, Kobayashi T, Hamapumbu H, Shields T, Mharakurwa S, Thuma PE, Louis TA, Glass G, Moss WJ: Reduced risk of malaria parasitemia following household screening and treatment: A cross-sectional and longitudinal cohort study. PLoS One 2012, 7:e31396.

19. Bousema T, Drakeley C: Epidemiology and Infectivity of Plasmodium falciparum and Plasmodium vivax gametocytes in relation to malaria control and elimination. Clin Microbiol Rev 2011, 24:377-410.

20. Moss WJ, Hamapumbu H, Kobayashi T, Shields T, Kamanga A, Clennon J, Mharakurwa S, Thuma PE, Glass G: Use of remote sensing to identify spatial risk factors for malaria in a region of declining transmission. Malar J 2011, 10:163 
21. Coleman RE, Kumpitak C, Ponlawat A, Maneechai N, Phunkitchar CV, Rachapaeq N, Zollner G, Sattabongkot J: Infectivity of asymptomatic Plasmodium-infected human populations to Anopheles dirus mosquitoes in western Thailand. J Med Entomol 2004, 41:201-208.

22. Poschl B, Waneesorn J, Thekisoe O, Chutipongvivate S, Panagiotis K: Comparative diagnosis of malaria infections by microscopy, nested PCR, and LAMP in Northern Thailand. AmJTrop Med Hyg 2010, 83:56-60.

23. McMorrow ML, Aidoo M, Kachur SP: Malaria rapid diagnostic tests in elimination settings -can they find the last parasite? Clin Microbiol Infect 2011, 17:1624-1631.

24. Schachterle SE, Ntove G, Levens JP, Clemens EG, Shi L, Raj A, Munoz B, Reller ME, West S, Dumler JS, Sullivan D: Prevalence and density-related concordance of three diagnostic tests for malaria in a region of Tanzania with hypoendemic malaria. J Clin Microbiol 2011, 49:3885-3891.

25. Mahajan B, Zheng H, Pham PT, Sedegah MY, Majam VF, Akolkar N, Rios M, Ankrah I, Madjitey P, Amoah G, Addison E, Quakyi IA, Kumar S: Polymerase chain reaction-based tests for pan-species and species-specific detection of human Plasmodium parasites. Transfusion 2012, doi:10.1111/j.15372995.2011.03541.x.

26. Shekalaghe SA, Drakeley C, van den Bosch S, ter Braak R, van den Bijllaardt W, Mwanziva C, Semvua S, Masokoto A, Mosha F, Teelen K, Hermsen R, Okell L, Gosling R, Sauerwein R, Bousema T: A cluster-randomized trial of mass drug administration with a gametocytocidal drug combination to interrupt malaria transmission in a low endemic area in Tanzania. Malar $\rfloor$ 2011, 10:247.

27. Greenwood BM: The micro epidemiology of malaria and its importance to malaria control. Trans R Soc Trop Med Hyg 1989, 83(Suppl):25-29.

28. Smith DL, Dushoff J, Snow RW, Hay Sl: The entomological inoculation rate and Plasmodium falciparum infection in African children. Nature 2005, 438:492-495.

29. WHO: Global malaria control and elimination: report of a technical review. World Health Organization; 2008.

doi:10.1186/1475-2875-11-353

Cite this article as: Stresman et al:: Malaria research challenges in low prevalence settings. Malaria Journal 2012 11:353.

\section{Submit your next manuscript to BioMed Central and take full advantage of:}

- Convenient online submission

- Thorough peer review

- No space constraints or color figure charges

- Immediate publication on acceptance

- Inclusion in PubMed, CAS, Scopus and Google Scholar

- Research which is freely available for redistribution 